\title{
ANALYTICAL BAND MODEL IN MONTE CARLO SIMULATION OF ELECTRIC TRANSPORT IN ZnS THIN FILM ELECTROLUMINESCENT DEVICES
}

\author{
H. ZHaO*, Y. WANG AND X. XU \\ Institute of Optoelectronic Technology, Northern Jiaotong University \\ Beijing 100044, China
}

(Received May 8, 2000)

\begin{abstract}
In this paper, an analytical band model is introduced in Monte Carlo simulation of electric transport process in thin film electroluminescent devices. The band structure of $\mathrm{ZnS}$ calculated from the empirical pseudopotential method is fitted by using polynomials. The density of states and scattering rates are also calculated from these polynomials. Based on these results, the electric transport process in ZnS-type thin film electroluminescent devices is simulated through the Monte Carlo method. By comparison with others, this model is as fast as the nonparabolic model and as accurate as the full band model. Furthermore, the influence of the band model on the simulation results is also investigated. We show that the dispersion relation and density of states are all important in the simulation.
\end{abstract}

PACS numbers: 78.60.-b, 72.20.-i

\section{Introduction}

Thin film electroluminescent (EL) devices have recently received much attention because of its application in flat panel displays [1]. The operation of EL devices requires the impact excitation of luminescent centers in a suitable host material by carrier accelerated to a sufficiently high energy. The exciting carriers must achieve an energy greater than the desired photon emission energy. As a result, the study of the high field transport properties of carriers in the host semiconductor material is of fundamental importance to the understanding and optimization of electroluminescent devices.

Since 1988 [2], several theoretical investigations have been made on high field carrier transport in $\mathrm{ZnS}$ by using Monte Carlo simulation. These investigations can be divided into three classes according to the models adopted to describe the band

*present address: Institute of Applied Physics, Karlsruhe University, 76128 Karlsruhe, Germany; e-mail: zhao@aphsrv1.physik.uni-karlssuhe.de 
structure of $\mathrm{ZnS}$. The first class uses the parabolic model [3, 4]. The results are unreasonable since this model is too simple to describe the real structure of bands. To overcome this problem, some researchers used the nonparabolic multivalley model to describe the band structure in their simulations $[5,6]$. We also studied several transport properties of $\mathrm{ZnS}$ by using this model [7-10]. Although this model is more accurate than the parabolic model, it is still different with the real structure, especially in a high-energy region. The most rigorous method is to use the full band structure gained from the empirical pseudopotential method (EPM) [11-15]. However, such a rigorous model requires enormous computational resources and, thus, is very difficult to be included in a device simulator.

Therefore, there is a need for a method that throws a bridge between the simple but inaccurate nonparabolic model and the rigorous but time consuming full band method. In order to keep the computational effort acceptable for simulation software used in the semiconductor device design and analysis, such a model should remain analytic. A possible way is to fit the real band structure calculated from EPM by polynomials. Then these polynomials are used in the simulations. In this method, the computation spends will approximate to that of the nonparabolic model since it remains analytic and the accuracy should approximate to that of the full band model since it describes the band structure correctly. In fact, this method has been attempted in $\mathrm{Si}[16,17]$ and GaAs [18], and is named as a "analytical band structure model". The results are reasonable. In this paper, we extend this method to $\mathrm{ZnS}$ and investigate the influence of the band model on the simulation results in detail.

\section{Fitting of the conduction band of $\mathrm{ZnS}$}

The Brillouin zone is divided into regions called extended valleys, as suggested by Mouton [18], in reference to the usual three-valley model. These regions are labeled $\Gamma 1, L 1$, and $X 1$ in the first conduction band. In the second conduction band, only $X 3$ valleys are considered for simplicity. For each region, we fit the dispersion relation by a two-piece polynomial function. One part starts from the minimum of the energy, the other one from the maximum

$$
\begin{aligned}
& k^{2}=a E+b E^{2}+c E^{3} \quad \text { when } \quad 0<k<k_{\mathrm{c}}, \\
& k^{\prime 2}=a E^{\prime}+b E^{\prime 2}+c E^{\prime 3} \text { when } k_{\mathrm{c}}<k<k_{\max },
\end{aligned}
$$

where $k^{\prime}=k_{\max }-k, E^{\prime}=E_{\max }-E ; k$ is the amplitude of the wave vector in the scale of $2 \pi / a_{0}$, where $a_{0}$ is the crystal constant of $\mathrm{ZnS} ; E$ is the energy in the scale of $\mathrm{eV} ; k_{\mathrm{c}}$ is the cross point of the two-piece polynomial; $a, b$, and $c$ are chosen in order to obtain the best fit for the dispersion relation. To account for anisotropy, the central valley $\Gamma 1$ is divided into three sectors corresponding to the crystallographic directions $\Lambda(\langle 111\rangle), \Delta(\langle 100\rangle)$, and $\Sigma(\langle 110\rangle)$. The other valleys are all divided into two sectors due to their two-direction anisotropy. Then the dispersion relation is fitted in each sector respectively.

Figure 1 shows the band-structure diagram of $\mathrm{ZnS}$ calculated with EPM [12] compared with our result. We can see that our result is in good agreement with that of EPM. We also draw the curves of the nonparabolic model in this figure. The error is obviously embedded in this model, especially in high-energy regions. 


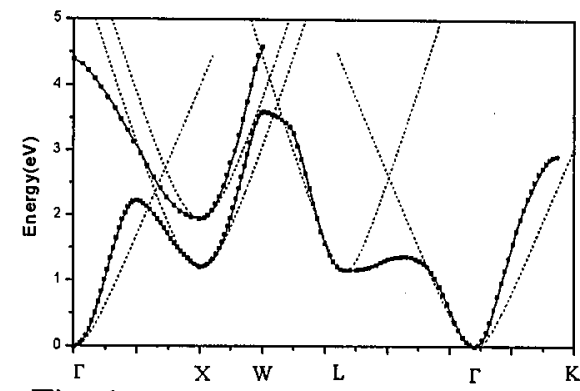

Fig. 1

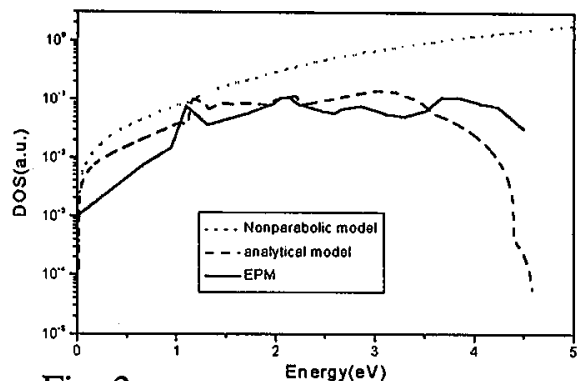

Fig. 2

Fig. 1. Conduction band structure of ZnS, calculated from: EPM [12] (solid line), the nonparabolic model (dashed line), the analytical model (points).

Fig. 2. Density of states in the conduction band of $\mathrm{ZnS}$.

Figure 2 shows the calculated density of states (DOS) in the conduction band of $\mathrm{ZnS}$ based on the above fitting results. DOS's calculated from the nonparabolic model and EPM [12] are also shown for comparison. According to this figure, DOS in the analytical band model shows a similar character to the EMP calculations. However, the consistency of these two curves is not as good as in Fig. 1. The reasons are as follows. In the analytical model, the fitting of the dispersion relations is only performed along some special directions, i.e., main axes in Brillouin zone. So, the points out of these axes may not be described very well by these polynomials. This may introduce errors in the calculation of DOS. In fact, this kind of discrepancy has also appeared when the analytical model was used in GaAs. Furthermore, the discrepancy of DOS in a high-energy region can be explained by the fact that we have neglected the third band. However, since only few electrons populate the third band, we think that this is sufficient for our simulations.

\section{Scattering rates}

To perform the simulation, the rates of each scattering mechanism must be known. The considered scattering mechanisms in our simulation include acoustic phonon scattering, polar optical phonon scattering, and intervalley scattering. We have calculated the rates of these scattering mechanisms in the nonparabolic model [10]. When the analytical model is adopted, the rates should be re-calculated. However, the band model influences the scattering rates mainly through the change of DOS [18]. So we can calculate the rates from the results of the nonparabolic model taking into account the difference of DOS between these two models. Let $N(E)$ and $N^{\prime}(E)$ denote DOS in the analytical model and the nonparabolic model, respectively. For a given scattering mechanism, the scattering rate $P^{\prime}(E)$ used in our simulation is simply deduced from the one, $P(E)$, calculated in the nonparabolic model by

$$
P^{\prime}(E)=\frac{N^{\prime}(E \pm \hbar \omega)}{N(E \pm \hbar \omega)} P(E)
$$

where $\hbar \omega$ is the energy exchanged in the scattering. 
Using this method, we calculate the rates of each scattering mechanism considered. The temperature is set to $300 \mathrm{~K}$ and the parameters used in these calculations are gained in Ref. [6]. Figure 3 shows the total rates of all mechanisms in different energies. Results of the nonparabolic model are also shown for comparison. Obviously, the rates gained in the analytical model are smaller than that of the nonparabolic model. This is reasonable, since in the analytical model, DOS is also smaller.

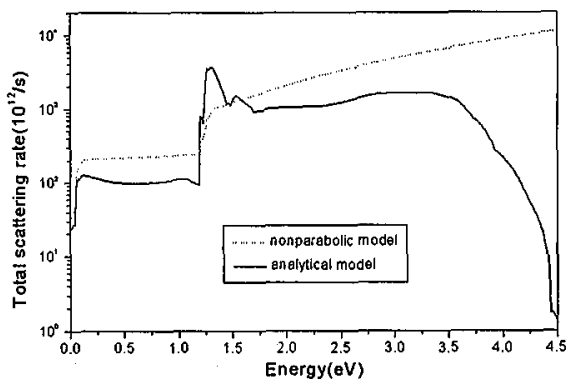

Fig. 3. Total scattering rate in $\mathrm{ZnS}$.

In EL devices, the operating electric field is very high. The band to band impact ionization is inevitable. In our simulation, we treat this effect as an additional scattering mechanism. The ionization rate is calculated as [19]

$$
P_{\text {io }}(E)=R\left(E-E_{\text {th }}\right)^{\alpha} \text { when } E>E_{\text {th }} \text {, }
$$

where $R=5.14 \times 10^{10} \mathrm{~s}^{-1}(\mathrm{eV})^{-\alpha}, E_{\mathrm{th}}=3.5 \mathrm{eV}$, and $\alpha=5.183$.

\section{Monte Carlo simulation}

Based on the above results, we simulated the electric transport process in ZnS-type film electroluminescent devices by using the analytical model. The details about the Monte Carlo method have been previously reported [7]. We also simulated this process in the same condition using the nonparabolic model for comparison. The computation times of the two simulations are almost the same.

The electron distribution function is the fundamental result of the Monte Carlo simulation, which allows one to derive all other quantities of interest. So, it is very important to check that the analytical model gives a reliable energy distribution function. Figures 4 and 5 show the distribution function gained in the analytical model under the electric field of $1 \mathrm{MV} / \mathrm{cm}$ and $2 \mathrm{MV} / \mathrm{cm}$, respectively. The results of the nonparabolic model and full band model [12] are also shown in these figures. According to these figures, the distribution functions gained in the analytical model coincide with that of the full band model. In fact, we have used a semi-logarithmic coordinate in these figures in order to show the difference between curves more clearly. In linear coordinate, the curves gained from these two models are almost the same. From this, we can conclude that the analytical model, which is based on the accurate fitting of the real band structure; is reasonable in the Monte Carlo simulation of the transport process in $\mathrm{ZnS}$. This conclusion 


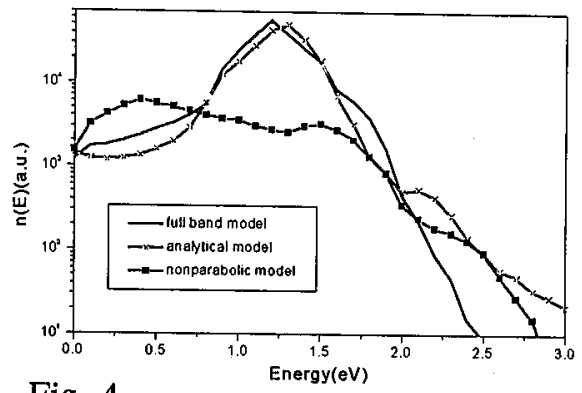

Fig. 4

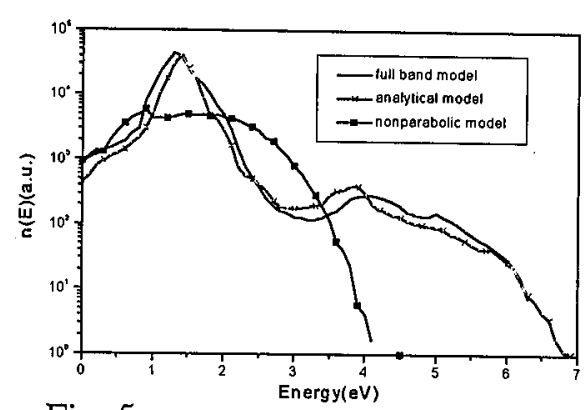

Fig. 5

Fig. 4. Electron energy distribution function in $\mathrm{ZnS}$ under the electric field of $1 \mathrm{MV} / \mathrm{cm}$. Fig. 5. Electron energy distribution function in $\mathrm{ZnS}$ under the electric field of $2 \mathrm{MV} / \mathrm{cm}$.

coincides with the previous attempts in $\mathrm{Si}$ and $\mathrm{GaAs}$ [16-18]. The curve gained in the nonparabolic model does not coincide with others in each figure, which shows the inaccuracy of the nonparabolic model.

\section{Influence of band model on simulation results}

The above results also prove the importance of band model adoption in simulations. It is interested to investigate the influence of the band model on the simulation process since this is a common problem in simulations of many kinds of material. In the Monte Carlo simulations, the band model influences the transport process in two ways. The one is to govern the energy gain of electron in each free flight. The other is to determine the rate of each scattering mechanism. In order to investigate these influences, we simulated the transport process in $\mathrm{ZnS}$ by using different band description methods. The calculated average energies under different electric fields $(0.5-5.0 \mathrm{MV} / \mathrm{cm})$ in each method are shown in Fig. 6. In these simulations, we do not only adopt the analytical and nonparabolic models, but also use the hybrid of these two models. That is, we use the scattering rates calculated from the analytical model but describe the free flight process using the nonparabolic model, or reversed. In this way, we can investigate each side of the influence separately. According to Fig. 6, the results of these four methods are different obviously. When the free flight and scattering rate are all described by the analytical model, the results coincide with that of the full band simulation [12].

Now we discuss the difference between these curves. In the free flight stage, the wave vector changes as

$$
\Delta k=k_{1}-k_{0}=F t .
$$

This is the same in all band models. But the change of energy, $\Delta E$, corresponding to this $\Delta k$ is determined by the dispersion relation. So, the energy gained in the free flight is different in every band model. Obviously, at the same free flight time, electrons will gain more energy in the band model in which the value of $\Delta E /|\Delta k|$ is higher. From Fig. 1, we can find that the dispersion relations of the nonparabolic model and analytical model are almost the same in a low-energy region. But in a high-energy region, the slope of the former is obviously higher than that of the latter. So, if we use the same scattering rates, the energy calculated from the 


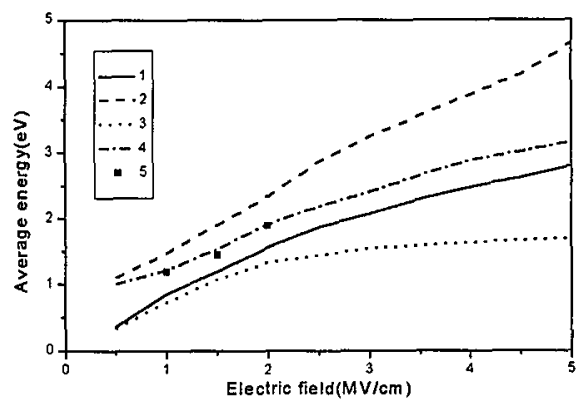

Fig. 6. Relations between average energy and electric field. The free flight and scattering are described by: 1 - both nonparabolic models, 2 - nonparabolic model and analytical model, 3 - analytical model and nonparabolic model, 4 - both analytical models.

simulation which used the nonparabolic model to describe the free flight stage should be higher than that used the analytical model. This can explain the fact that curve 1 is higher than curve 3 and curve 2 is higher than curve 4 in Fig. 6.

The scattering rates calculated from the nonparabolic model are higher than that from the analytical model since DOS is overrated in the nonparabolic model. That means electrons will suffer more scattering events if we use the nonparabolic model to describe the scattering process. So, if we use the same model to describe the free flight stage but a different model, nonparabolic model or analytical model, to describe the scattering process, the former will result in a lower energy than the latter. This is the reason that curve 2 is higher than curve 1 and curve 4 is higher than curve 3 in Fig. 6.

Compared with the analytical model, the nonparabolic model overrates the energy gained in each free flight, which induced in a higher calculated energy. At the same time, it overrates the scattering rates, which induced in a lower calculated energy. So, as the comparison of curve 1 and curve 4 in Fig. 6 , we cannot make the judgment simply as the above qualitative discussions. Based on our simulations, curve 4 is higher than curve 1 . That is, the nonparabolic model underrates the average energy of electrons in the Monte Carlo simulations.

We have discussed the influence of the band model on the simulation results. Fischetti and Laux [20] have proposed that the influence of the band model on the simulation results is given mainly by the density of states and the dispersion relation is less important. Based on this idea, Vogelsang and Hansch [21] set up a model for the Monte Carlo simulation of the electric transport in silicon. In this model, they derived the energy-dependent effective mass and the dispersion relation directly from DOS calculated from EPM. This means that the influence of real dispersion relation on the free flight stage was neglected at all. The results of this model does not coincide exactly with that of the full band models. According to our simulations, at least in $\mathrm{ZnS}$, this idea is not reasonable. The dispersion relation and DOS are all important in the Monte Carlo simulations. 


\section{Conclusion}

The analytical band model is introduced in the Monte Carlo simulation of thin film electroluminescent devices in this paper. This model is based on the accurate fitting of the dispersion relation calculated from EPM by two-piece polynomials. DOS is also reproduced from these polynomials. The scattering rate is calculated and compared with the nonparabolic model. According to our simulations, this model yields electron distribution functions very similar to those obtained by the most rigorous full band models. Furthermore, the calculation time and the computer requirement are almost the same as the nonparabolic model and far less than that of the full band model. So, this model could throw a bridge between the simple and rapid but inaccurate nonparabolic model and the rigorous but time consuming full band model. This model could be used to develop high-efficiency device simulation software. Furthermore, it may be adapted to other II-VI materials, with very few modifications. In this paper, we also investigated the influence of the band model on the simulation results in detail. We found that the DOS and dispersion relation are all important in the simulations. So, the inaccuracy in any of them will introduce error in simulation results.

\section{Acknowledgement}

This work is supported by National Nature Science Foundation of China and Paper Foundation of NJTU. One of us (Y.W.) acknowledges the support of the stride-century foundation of Chinese Education Ministry.

\section{References}

[1] Y.A. Ono, Electroluminescent Displays, World Scientific, Singapore 1995, p. 3.

[2] K. Brennan, J. Appl. Phys. 64, 4024 (1988).

[3] R. Mach, G.O. Muller, J. Cryst. Growth 101, 967 (1990).

[4] H.J. Fitting, J. Cryst. Growth 101, 876 (1990).

[5] K. Bhattacharyya, S.M. Goodnick, J.F. Wager, J. Appl. Phys. 73, 3390 (1993).

[6] J. Fogarty, W. Kong, R. Solanki, Solid-State Electronics 38, 653 (1995).

[7] H. Zhao, Y.S. Wang, Z. Xu, X.R. Xu, J. Phys., Condens. Matter 11, 2145 (1999).

[8] H. Zhao, Y.S. Wang, Z. Xu, X.R. Xu, Semicond. Sci. Technol. 14, 1098 (1999).

[9] H. Zhao, Y.S. Wang, Z. Xu, X.R. Xu, Acta Phys. Pol. A 96, 475 (1999).

[10] H. Zhao, Y.S. Wang, Z. Xu, X.R. Xu, Science in China E 42, 282 (1999).

[11] S.M. Goodnick, M. Duer, S. Pennathur, Inorganic and Organic Electroluminescence, World Scientific, Singapore 1996, p. 13.

[12] M. Duer, S.M. Goodnick, S. Pennathur, J.F. Wager, M. Reigrotzki, R. Redmer, J. Appl. Phys. 83, 3176 (1998).

[13] E. Bellotti, K. Brennan, R. Wang, P.P. Ruden, J. Appl. Phys. 83, 4765 (1998).

[14] I. Lee, S. Pennathur, S.M. Goodnick, J.F. Wager, J. Korean Phys. Soc. 31, 517 (1997).

[15] M.L. Cohen, J.R. Chelikowsky, Electronic Structure and Optical Properties of Semiconductor, Springer, New York 1988, p. 112. 
[16] R. Brunetti, C. Jacoboni, F. Venturi, E. Sangiorgi, B. Ricco, Solid State Electronics 32, 1663 (1989).

[17] X. Wang, V. Chandramouli, C.M. Maziar, A.F. Tasch, J. Appl. Phys. 73, 3339 (1993).

[18] O. Mouton, J.L. Thobel, R. Fauquembergue, J. Appl. Phys. 81, 3160 (1991).

[19] M. Reigrotzki, M. Stobbe, R. Redmer, Phys. Rev. B 52, 1456 (1995).

[20] M. Fischetti, S. Laux, Phys, Rev. B 38, 9721 (1988).

[21] T. Vogelsang, W. Hansch, J. App. Phys. 70, 1493 (1991). 